\title{
False-Name-Proofness in Social Networks
}

\author{
Vincent Conitzer \\ Duke University \\ Durham, NC, USA \\ conitzer@cs.duke.edu
}

\author{
Nicole Immorlica \\ Northwestern University \\ Evanston, IL, USA \\ nicimm@gmail.com
}

\author{
Joshua Letchford \\ Duke University \\ Durham, NC, USA \\ jcl@cs.duke.edu
}

\author{
Kamesh Munagala \\ Duke University \\ Durham, NC, USA \\ munagala@cs.duke.edu
}

\author{
Liad Wagman \\ Illinois Institute of Technology \\ Chicago, IL, USA \\ wagman@stuart.iit.edu
}

\begin{abstract}
In mechanism design, the goal is to create rules for making a decision based on the preferences of multiple parties (agents), while taking into account that agents may behave strategically. An emerging phenomenon is to run such mechanisms on a social network; for example, Facebook recently allowed its users to vote on its future terms of use. One significant complication for such mechanisms is that it may be possible for a user to participate multiple times by creating multiple identities. Prior work has investigated the design of false-name-proof mechanisms, which guarantee that there is no incentive to use additional identifiers. Arguably, this work has produced mostly negative results. In this paper, we show that it is in fact possible to create good mechanisms that are robust to false-name-manipulation, by taking the social network structure into account. The basic idea is to exclude agents that are separated from trusted nodes by small vertex cuts. We provide key results on the correctness, optimality, and computational tractability of this approach.
\end{abstract}

\section{Introduction}

Recently, Facebook, Inc. decided to allow its users to vote on its future terms of use [19]. While the result was not binding, ${ }^{1}$ this vote represents a new phenomenon that is likely to become more prominent in the future: agents participating in an election or other mechanism through a social networking site. Holding an election among the users of a social networking site introduces some issues that do not appear in regular elections. Perhaps the foremost such issue, and the one that we will focus on, is that it is generally easy for a user to create additional accounts/identities, allowing her to vote multiple times. This can compromise the legitimacy of the election and result in a suboptimal alternative being chosen.

The topic of designing elections or other mechanisms for settings where it is easy to create multiple identities and participate multiple times has already received some attention. The primary approach has been to design mechanisms that are false-name-proof $[15,16]$, meaning that an agent never benefits from participating more than once. (This is analogous to the better-known concept of strategy-proofness, meaning that an agent never benefits from misreporting her preferences. In fact, false-name-proofness is often defined in a way that subsumes strategy-proofness.) Unfortunately, existing results on false-name-proofness are quite negative, especially in voting contexts. For the case where additional identities can be created at zero cost, a general

\footnotetext{
${ }^{1}$ The result would have been binding if at least $30 \%$ of all active users had voted, a seemingly impossibly high turnout in this context.
} 
characterization of false-name-proof voting mechanisms has been given [5]; this characterization implies that for the special case where there are only two alternatives, the best we can do is the unanimity mechanism. This mechanism works as follows: if all voters agree on which alternative is better, that alternative is chosen; but if there is any disagreement (no matter in which proportions), then a fair coin is flipped to decide between the alternatives. This is an extremely negative result, since the mechanism is almost completely unresponsive to the votes. ${ }^{2}$ Several ways to circumvent such negative results have been proposed, such as assuming that creating additional identities comes at a small cost [14] or considering a model in which it is possible to verify some of the identities [4].

These prior results do not consider any social network structure that may hold among the identities. Rather, these earlier results can be thought of as applying to settings where a user creates an account for the sole purpose of casting a vote (or bid, etc.), so that no social network structure is specified. We will show in this paper that by using the social network structure in the mechanism, it is possible to obtain much more positive results, because fake identities will look suspect in the social network (graph) structure. To give some intuition, consider John Doe, who has a legitimate account on the social networking site. In order to cast more votes, he can create several other identities (false names), such as Jane Jones and Jimmy Smith. Among the accounts that he controls, he can create any network structure by linking them to each other. However, if the other users behave legitimately, then he will not be able to link his additional accounts to any of the other users' identities (since, after all, they have never heard of Jane Jones or Jimmy Smith); he will only be able to get his friends to link to his legitimate identity (John Doe). This results in an odd-looking social network structure, where his legitimate identity constitutes a vertex cut in the graph, whose removal separates the fake identities from the rest of the graph.

In the remainder of this paper, we generalize the intuition afforded in the above scenario, giving a notion of when a node is "suspect" based on small vertex cuts that separate it from the trusted nodes. In Section 2, we formally define the setting we will be focusing on. In Section 3, we discuss false-name-proofness and provide a sufficient condition for guaranteeing it. In Section 4, we discuss how to find all suspect nodes when trusted nodes are given exogenously to the algorithm. Then, in Section 5, we extend our analysis to settings in which we do not have trusted nodes initially, but we can actively verify nodes. We give both correctness and optimality results. Finally, in Appendix B, we give experimental results on simulated social networks for the techniques in Section 4 and Section 5.

\subsection{Related work}

The basic intuition that the creation of false identities in a social network results in suspiciously small vertex cuts has previously been explored in several papers, in the context of peer-to-peer networks $[18,17]$ and web spam detection $[2,3,6,7,13]$.

The work on fraud in peer-to-peer networks attempts to thwart sybil attacks in which one or more malicious users obtain multiple identities in order to out-vote legitimate users in collaborative tasks like Byzantine failure defenses. These papers propose protocols that ensure that not too many false identities are accepted. While this may be sufficient to thwart certain sybil attacks in decentralized distributed systems, it can still leave incentives for an agent to create multiple identities, especially in applications such as elections in which the electorate is about evenly divided. Furthermore, a major hurdle in the sybil attack research is that any protocol must be decentralized. In contrast, in this paper, we follow the stricter approach of guaranteeing that the creation of false identities is always weakly suboptimal, corresponding to the standard approach in the mechanism design literature. On the other hand, we allow our mechanisms to be centralized, as we envision

\footnotetext{
${ }^{2}$ The literature on false-name-proof voting mechanisms is quite recent: earlier work on false-name proofness considered other settings, such as combinatorial auction mechanisms, where multiple items are for sale at the same time. Unfortunately, here, too, there are strong impossibility results, including a result that states that under certain conditions, from the perspective of a worst-case efficiency ratio, it is impossible to significantly outperform the simple mechanism that sells all items as a single bundle [8].
} 
them being run by the proprietor of the social network who has access to the network structure.

Fraud is also prevalent in the world wide web where users sometimes create fake webpages and links with the sole intent of boosting the PageRank of given website(s). Several researchers have considered using link structure to combat spam [2, 3, 6, 7, 13]. In SpamRank [2, 3], the authors assume a node is suspect if the main contribution to its PageRank is generated from a small set of supporting nodes (see also [6]). Our focus on small vertex cuts can be interpreted as an extreme version of the conditions proposed in SpamRank. An alternative approach, as taken by TrustRank [7] and Anti-TrustRank [13], assumes the existence of an oracle (e.g., a human being) which is able to determine the legitimacy of any given website. Calls to the oracle are, however, expensive, and so the main task in the protocol is to select a seed set of pages. The protocol then guesses the legitimacy of the remaining pages based on their connectivity to the seed set. In particular, the protocol assumes that legitimate pages rarely point to illegitimate ones, and hence the illegitimate pages are those that are "approximately isolated." Again, this approach is similar to our approach at a high level; the selection of the seed set corresponds to our verification policy (discussed later in the paper), and the condition of approximate isolation corresponds to the condition of small vertex cuts in our work. Despite these similarities, the particulars of the model and definitions are quite different, as these existing protocols are designed to combat fraudulent attacks in PageRank, whereas our goal is to prevent fraudulent attacks in voting or other mechanisms.

\section{Setting}

Our results can be applied to any mechanism design domain, but for the sake of concreteness, it may be helpful to think about the simple setting in which $m$ agents must select between two alternatives. Each agent has a strict preference for one alternative over the other. The mechanism designer wishes to make a socially desirable choice, i.e., select an alternative that is beneficial for society as a whole. The majority rule, in which the alternative preferred by more voters wins, would be ideal; unfortunately, the majority rule will result in incentives to create false names, if naïvely applied.

Agents are arranged in a social network consisting of $n$ nodes where $m \leq n$. Each agent $i$ has a legitimate account in the social network, corresponding to a node $v_{i}^{t}$, as well as a (possibly empty) set of illegitimate accounts $V_{i}^{f}$. There is an arbitrary graph structure among the legitimate nodes in the social network-that is, we impose no structure on the subgraph induced by the legitimate nodes $\left\{v_{i}^{t}\right\}_{i \in\{1, \ldots, m\}}$.

In the most basic version of our model, we assume that no two manipulating agents can work together, so that an agent can only link her illegitmate nodes to each other and to her own legitimate node. Hence, for any $i \neq j$, there are no edges between $V_{i}^{f}$ and $\left\{v_{j}^{t}\right\} \cup V_{j}^{f}$. However, for each agent $i$, we allow an arbitrary graph structure on the set of nodes $\left\{v_{i}^{t}\right\} \cup V_{i}^{f}$.

In the more general version of our model, we assume that up to $k$ agents can collude together. (The basic model is the special case where $k=1$.) That is, the agents $1, \ldots, m$ are partitioned into coalitions $S_{j} \subseteq\{1, \ldots, m\}$, with $\left|S_{j}\right| \leq k$ for each $j$. Let $V_{S_{j}}^{f}$ be the set of all illegitimate nodes used by $S_{j}$, that is, $V_{S_{j}}^{f}=\bigcup_{i \in S_{j}} V_{i}^{f}$, and let $V_{S_{j}}^{t}$ be the set of all legitimate nodes used by $S_{j}$, that is, $V_{S_{j}}^{t}=\bigcup_{i \in S_{j}}\left\{v_{i}^{t}\right\}$. Two distinct coalitions cannot link their illegitimate nodes to each other, so that for any $i \neq j$, there are no edges between $V_{S_{i}}^{f}$ and $V_{S_{j}}^{t} \cup V_{S_{j}}^{f}$. However, for each coalition $S_{i}$, we allow an arbitrary graph structure on the set of nodes $V_{S_{i}}^{t} \cup V_{S_{i}}^{f}$.

To summarize, our social network setting consists of

- a set of $m$ agents denoted $\{1, \ldots, m\}$,

- a set of $m$ legitimate nodes, one for each agent, denoted $V^{t}=\left\{v_{1}^{t}, \ldots, v_{m}^{t}\right\}$,

- a collection of $m$ (possibly empty) sets of illegitimate nodes, one for each agent, denoted $\left\{V_{1}^{f}, \ldots, V_{m}^{f}\right\}$, 
- a partition of the agents $\{1, \ldots, m\}$ into subsets $S_{j}$, where $\left|S_{j}\right| \leq k$ (the no-collusion case corresponds to $k=1$ ), such that for any $i, j$, there are no edges between $V_{S_{i}}^{f}$ and $V_{S_{j}}^{t} \cup V_{S_{j}}^{f}$ (apart from this, the graph structure can be arbitrary).

Some of the nodes in the graph will be trusted. For example, the mechanism designer may personally know the agents corresponding to these nodes in the real world. This is a case in which trust is exogenous, that is, we have no control over which agents are trusted: the trusted agents are given as part of the input. Later in the paper, we will consider settings where we can, with some effort, verify whether any particular node is legitimate (for example, by asking the node for information that confirms that there is a corresponding agent in the real world). Nodes that pass this verification step become trusted nodes; this is a case of endogenous trust. It should be noted that, in either case, we do not assume that a trusted node will refrain from creating additional identifiers. That is, the only sense in which the node is trusted is that we know it corresponds to a real agent.

The mechanisms that we consider in this paper operate as follows. A suspicion policy is a function that takes as input the social network graph $G=(V, E)$ as well as a set $T$ of trusted nodes, $T \subseteq V^{t} \subseteq V$; and as output labels every node in $V$ as either "deemed legitimate" or "suspect." Generally, all the nodes in $T$ will be deemed legitimate, but others may be deemed legitimate as well based on the network structure. Subsequently, all the nodes that have been deemed legitimate get to participate (e.g., vote) in a standard mechanism $f$ (e.g., the majority rule), and based on this an outcome is chosen. (In this context, we only consider anonymous mechanisms that treat all nodes that get to participate identically.) In the case where nodes become trusted through verification, we also have a verification policy that takes $G$ as input and, based on this, determines which nodes to verify.

We consider a game played between the mechanism designer and the agents (more precisely, the coalitions $S_{j}$ ). First, the mechanism designer announces her mechanism, consisting of $f$ and the suspicion policy (and, in the case where trust is obtained through verification, a verification policy). Then, each coalition $S_{j}$ creates its illegitimate nodes $V_{S_{j}}^{f}$, as well as the edges that include these nodes (they can only have edges to other nodes in $V_{S_{j}}^{f}$, and to $V_{S_{j}}^{t}$ ). Note that the coalitions do not strategically determine edges between legitimate nodes in this game: in order to focus on false-name manipulation, only the creation of false nodes and their edges is modeled in the game. Also note that the mechanism designer, when announcing her mechanism, is unaware of the true graph as well as which agents are in coalitions together.

After obtaining the social network graph (and, possibly, some exogenously trusted nodes), the mechanism designer runs (1) (possibly) the verification policy and (2) the suspicion policy. The designer subsequently asks the nodes that have been deemed legitimate to report their preferences, and then finally runs (3) the standard mechanism $f$ on these reported preferences, to obtain the outcome.

Whether this results in incentives for using false names depends on all of the components (1), (2), and (3), and each one individually can be used to make the whole mechanism false-name-proof. For example (for component 3 ), if $f$ is by itself false-name-proof, then even if we verify no nodes and deem every node legitimate, there is still no incentive to engage in false-name manipulation. The downside of this approach is that we run into all the impossibility results from the literature on designing false-name-proof mechanisms. Similarly (for component 1), if we verify all nodes and then only deem the trusted nodes (the ones that passed the verification step) legitimate, there is no incentive to use false names. Of course, this generally results in far too much overhead. In this paper, we will be interested in suspicion policies (component 2) that by themselves guarantee that there is no incentive to use false names. For this, we heavily rely on the social network structure. In the first part of the paper, we do not consider verification policies-we take which nodes are trusted as given exogenously. 


\section{False-name-proofness}

To define what it means for a suspicion policy to guarantee false-name-proofness, we first need to define some other properties. The next two definitions assume that a coalition can be thought of as a single player with coherent preferences; this is reasonable in the sense that if there is internal disagreement within the coalition, this will only make it more difficult for them to manipulate the mechanism.

Definition 1 A standard mechanism $f$ is $k$-strategy-proof if it is a dominant strategy for every coalition of size at most $k$ to report truthfully.

Definition 2 A standard (anonymous) mechanism $f$ satisfies $k$-voluntary participation if it never helps a coalition of size at most $k$ to use fewer identifiers.

Because the coalitions play a game with multiple stages, it is important to specify what we assume the coalitions learn about each other's actions in earlier stages - that is, what are the information sets in the extensive form of the game? Specifically, when a coalition reports its preferences to $f$, what does the coalition know about the nodes and edges created by other coalitions? We assume that a coalition learns nothing about other coalitions' actions, except that the coalition can (possibly) make inferences about what others have done based on which of its own nodes have been deemed legitimate. Thus, it is assumed that each coalition is rational and has perfect recall, but also that it does not have any other way of observing what other coalitions have done. The following makes this precise.

Definition 3 We say that the Limited Information Assumption (LIA) holds if, for every coalition $S_{j}$, for every two nodes ${ }^{3} \nu_{1}, \nu_{2}$ in the extensive form of the game (where $S_{j}$ is about to report preferences to $f$ ), the following holds. If $S_{j}$ has taken the same node-and-edge creation actions at $\nu_{1}$ and $\nu_{2}$, and the same nodes have been deemed legitimate for $S_{j}$ at $\nu_{1}$ and $\nu_{2}$, then these nodes are in the same information set-that is, $S_{j}$ cannot distinguish them.

It should be emphasized that LIA does not specify the information sets exactly-it is merely an upper bound on how much the coalitions learn about each other's actions. Specifically, we can also require the coalitions to report preferences for nodes before informing them exactly which of these nodes have been deemed legitimate. In an extreme special case of this (for which our results still hold), we can consider the situation where a coalition must create nodes and edges and report preferences for its nodes at the same time, making the game a single-stage game. In this case, when a coalition is reporting preferences, it clearly knows nothing about what the other coalitions have done at all, since they are moving at the same time. This is equivalent to saying that a coalition first creates nodes and edges, and then reports preferences for these nodes but without learning anything (including which of these nodes have been deemed legitimate). This is consistent with LIA; it just means that even more nodes in the game tree are in the same information set than is strictly required by LIA. Of course, it is more elegant and efficient from a communication point of view to only ask for the preferences of the nodes that have been deemed legitimate, and as we will see, this does not cause problems with incentives for the techniques we propose in this paper.

We now define what it means for a suspicion policy to guarantee false-name-proofness.

Definition 4 A suspicion policy $\Pi$ guarantees false-name-proofness for coalitions of size at most $k$ if, under the LIA assumption, the following holds. for any standard (anonymous) mechanism $f$ that is $k$-strategy-proof and satisfies $k$-voluntary participation, if we combine $\Pi$ with $f$, then for any true social network structure on $V^{t}$, for any initial trusted nodes $T \subseteq V^{t}$, and for any partition of $V^{t}$ into coalitions $S_{j}$ of size at most $k$ each, it is a dominant strategy for each coalition to set $V_{S_{j}}^{f}=\emptyset$ and report truthfully.

\footnotetext{
${ }^{3}$ These are not to be confused with the nodes in the network.
} 


\subsection{A sufficient condition for guaranteeing false-name-proofness}

We now provide a sufficient condition for guaranteeing false-name-proofness, with which we will work in the rest of the paper.

Definition 5 A suspicion policy $\Pi$ is $k$-robust if, for any true social network structure on $V^{t}$, for any initial trusted nodes $T \subseteq V^{t}$, and for any partition of $V^{t}$ into coalitions $S_{j}$ of size at most $k$ each, we have the following. For every coalition $S_{j}$, for every profile of actions taken by the other coalitions:

1. The actions of $S_{j}$ (in terms of creating new nodes and edges) do not affect which of the other coalitions' identifiers $\left(V \backslash\left(V_{S_{j}}^{t} \cup V_{S_{j}}^{f}\right)\right)$ are deemed legitimate.

2. The number of identifiers in $V_{S_{j}}^{t} \cup V_{S_{j}}^{f}$ that are deemed legitimate is maximized by setting $V_{S_{j}}^{f}=\emptyset$.

Theorem 1 If a suspicion policy $\Pi$ is $k$-robust, then it guarantees false-name-proofness for coalitions of size at most $k$.

All the proofs have been moved to Appendix A due to the space constraint.

\section{Exogenously Given Trusted Nodes}

We begin by studying the case where the trusted nodes $T$ are given exogenously. This could correspond to the case where the mechanism designer personally knows the owners of some of the nodes on the network, or perhaps these nodes have already been successfully verified in an earlier stage. Later in the paper, we will study the case where there are no exogenously given trusted nodes, so that we have to decide which nodes to verify. Given $G$ and $T$, the next step is to determine which nodes to label as "suspect," based on the fact that they are not well connected to trusted nodes. We will make our suspicion policy precise shortly, but first we illustrate the basic idea on a small example. We recall that $k$ denotes the maximum size of a coalition of colluding agents. Figure 1 gives an example of a network with two exogenously given trusted nodes, for the case where $k=1$. As the figure illustrates, nodes that are separated from the trusted nodes by a vertex cut of size 1 could be false identities created by the node on the vertex cut in order to manipulate the outcome of the mechanism. Hence, they are deemed suspect.

In the following subsections, we first define our suspicion policy precisely and prove that it has several nice properties, including guaranteeing false-name-proofness. We then prove that this policy is optimal in the sense that any other suspicion policy with these properties would label more nodes as suspect. Finally, we give a polynomial-time algorithm for determining whether nodes are deemed legitimate or suspect under this policy. In Appendix B.1 we test these ideas on some simulated social networks.

\subsection{The suspicion policy}

One natural approach is to label as suspect every node $v$ that is separated from all the trusted nodes by a vertex cut of size at most $k$ (this cut may include some of the trusted nodes). After all, such a node $v$ may have been artificially created by the coalition of nodes corresponding to its vertex cut. On the other hand, for a node $v$ that is not separated from the trusted nodes by any vertex cut of size at most $k$, there is no coalition of nodes that could have artificially created $v$. While this reasoning is correct, it turns out that, to guarantee false-name-proofness, it is not sufficient to label only the nodes separated from the trusted nodes by a vertex cut of size at most $k$ as suspect. The reason is that this approach may still leave an incentive for a coalition to create false nodes: not because these false nodes will be deemed legitimate, but rather because it may prevent other nodes from being labeled as suspect. We first observe a fundamental property of nodes being separated from the trusted nodes by a vertex cut of size at most $k$. 


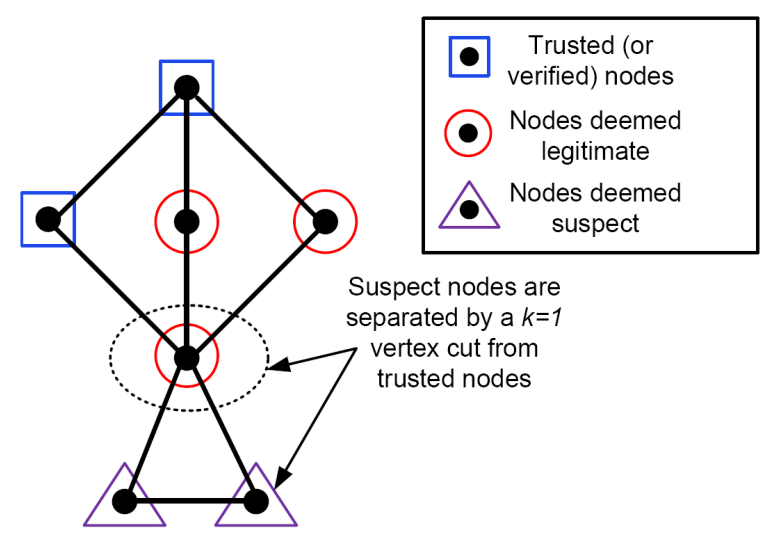

Figure 1: Example network. The nodes correspond to identities (user accounts), and the edges correspond to (say) friendship relations between the identities. The mechanism designer, at this point for exogenous reasons, considers certain nodes "trusted" (marked by squares), that is, she is sure that they are not false names. The nodes marked with triangles are separated from the trusted nodes by a vertex cut of size one (indicated by the dotted ellipse). As a result, it is conceivable that these nodes are false names, created by the agent corresponding to the vertex-cut node; hence, they are labeled suspect. The remaining nodes are not separated from the trusted nodes by a vertex cut of size one, and as a result they are deemed legitimate (marked by circles).

Lemma 1 For an initially untrusted node $v$, the following two statements are equivalent.

1. $v$ is not separated from the initially trusted nodes by a vertex cut of size at most $k$ (which may include initially trusted nodes).

2. There exist $k+1$ vertex-disjoint paths from (distinct) initially trusted nodes to $v$.

The problem with the approach above is that a coalition may use false nodes that will be labeled suspect, but that help create paths to other nodes that will be deemed legitimate as a result. The solution is to apply the procedure iteratively, in each stage removing the nodes that are separated from all the trusted nodes by a vertex cut of size at most $k$, until convergence. An example where this is necessary appears in Figure 2 in Appendix A.

Definition 6 Let $r$ take as input $G=(V, E)$ and $T \subseteq V$, and as output produce the subgraph $G^{\prime}$ of $G$ that results from removing those nodes in $V-T$ that are separated from the trusted nodes $T$ by a vertex cut of size at most $k$ (as well as removing the edges associated with these nodes). These vertex cuts are allowed to include nodes in T. Let $G=G^{(0)}, G^{\prime}=G^{(1)}, G^{(2)}, \ldots, G^{\left(n_{G, T}\right)}$ be the sequence of graphs that results from applying $r$ iteratively on $\left(G^{(i)}, T\right)$, where $n_{G, T}$ is the smallest number satisfying $G^{\left(n_{G, T}\right)}=G^{\left(n_{G, T}-1\right)}$ (note this sequence must converge as the set of nodes in successive iterations is nonincreasing and nonnegative). Then our suspicion policy $\Pi_{k}^{*}$, when applied to $(G, T)$, deems all the nodes in $G^{\left(n_{G, T}\right)}$ legitimate, and all the other nodes in $G$ suspect.

In each iteration, the procedure for computing $\Pi_{k}^{*}$ removes all the nodes that are at that point separated from all the trusted nodes by a vertex cut of size at most $k$. This corresponds to eliminating nodes in a 
particular order. One may wonder if the result would be any different if we eliminated nodes in a different order, for example, in one iteration removing only a subset of the nodes that are at that point separated from all the trusted nodes by a vertex cut of size at most $k$, before continuing to the next iteration. This is analogous to the notion of path independence of iterated strict dominance in game theory: no matter in which order we eliminate strictly dominated strategies, in the end we obtain the same set of remaining strategies [10]. (This is in contrast to iterated weak dominance, where the order of elimination does affect the final remaining strategies.) We will show a similar path independence result for removing nodes in our setting. To do so, we first define the class of suspicion policies that correspond to some order; then we show that the class has only one element, namely, $\Pi_{k}^{*} \cdot{ }^{4}$

Definition 7 Let $\Pi_{k}$ be the class of all suspicion policies that correspond to a procedure where:

- In each iteration, some subset of the nodes that are at that point separated from all the trusted nodes by a vertex cut of size at most $k$ is eliminated from the graph;

- This subset must be nonempty when possible;

- When no additional nodes can be eliminated, the remaining nodes are exactly the ones deemed legitimate.

Lemma 2 The class $\Pi_{k}$ consists of a singleton element $\Pi_{k}^{*}$, i.e., $\Pi_{k}=\left\{\Pi_{k}^{*}\right\}$.

We now show that our policy $\Pi_{k}^{*}$ guarantees false-name-proofness for coalitions of size at most $k$.

Lemma 3 Let $G=(V, E)$ be a graph and let $T \subseteq V$ be the trusted nodes. Let $G^{\prime}$ be a graph that is obtained from $G$ by adding additional nodes $V^{\prime}$ and additional edges $E^{\prime}$ that each have at least one endpoint in $V^{\prime}$-in such a way that every node in $V^{\prime}$ is separated from $T$ by a vertex cut of size at most $k$. Then, applying $\Pi_{k}^{*}$ to $G^{\prime}=\left(V \cup V^{\prime}, E \cup E^{\prime}\right)$ and $T$ results in the same nodes being deemed legitimate as applying $\Pi_{k}^{*}$ to $G$ and $T$.

Theorem $2 \Pi_{k}^{*}$ is $k$-robust (and hence, by Theorem 1, guarantees false-name-proofness for coalitions of size at most $k$ ). Moreover, under $\Pi_{k}^{*}$, a coalition $S_{j}$ 's actions also do not affect which of its own legitimate nodes $V_{S_{j}}^{t}$ are deemed legitimate. Finally, $\Pi_{k}^{*}$ is guaranteed to label every illegitimate node as suspect.

\subsection{Optimality}

We now show that $\Pi_{k}^{*}$ is the best possible suspicion policy in the sense that any other policy satisfying the desirable properties in Theorem 2 must label more nodes as suspect.

Theorem 3 Let $\Pi^{\prime}$ be a suspicion policy that (1) is $k$-robust, (2) is such that a coalition $S_{j}$ 's actions also do not affect which of its own legitimate nodes $V_{S_{j}}^{t}$ are deemed legitimate, and (3) is guaranteed to label every illegitimate node as suspect. Then, if $\Pi_{k}^{*}$ labels a node as suspect, then so must $\Pi^{\prime}$.

The next subsection shows that we can run $\Pi^{*}$ in polynomial time.

\footnotetext{
${ }^{4}$ The different orders of course correspond to different procedures for computing which nodes are deemed legitimate, but we will show that as a function that determines which nodes are finally deemed legitimate, they are all the same.
} 


\subsection{Polynomial-time algorithm for determining whether a node is trusted}

In this subsection, we give a polynomial-time algorithm for determining whether nodes are deemed legitimate or suspect according to $\Pi_{k}^{*}$. The key step is to find an algorithm for figuring out which nodes are separated from the trusted nodes by a vertex cut of size at most $k$; then we can simply iterate this in order to execute $\Pi_{k}^{*}$ (and by Lemma 2 we do not need to be careful about the order in which we eliminate nodes). It turns out that by Lemma 1, we can do this by solving a sequence of maximum flow problem instances.

Theorem 4 Given $G=(V, E)$ and $T \subseteq V$, we can determine in polynomial time which nodes are not separated from $T$ by a vertex cut of size at most $k$. As the number of iterations of $\Pi_{k}^{*}$ is bounded by $|V|$, we can run $\Pi_{k}^{*}$ in polynomial time.

\section{Choosing Nodes to Verify (Endogenous Trust)}

Our methodology requires some nodes to be trusted. So far, we have considered settings where some nodes are trusted for exogenous reasons (for example, the organizer's own friends may be the only trusted nodes). However, we can also endogenize which nodes are trusted, by assuming that the organizer can invest some effort in verifying some of the identities to establish their legitimacy (for example, by asking these identities for information that identifies them in the real world). This is an approach that has been considered before in the context of false-name-proofness [4], but that prior work paid no regard to social network structure. The social network structure can drastically reduce the amount of verification required, because, as we have seen earlier in this paper, once we have some nodes that are trusted, we can infer that others are legitimate.

There are (at least) two approaches to consider here: verify enough nodes so that no suspect nodes remain at all (and try to minimize the number of verified nodes under this constraint), or try to maximize the number of nodes deemed legitimate, given a budget of verifications (say, at most $r$ verifications). In this paper, we focus on the former.

Technically, a verification policy consists of a contingency plan, where the next node to verify depends on the results of earlier verifications of nodes (which can either fail or succeed). If a verification fails, that node is classified as illegitimate, and the verification continues. The verification continues until no nodes remain suspect (other than ones that failed the verification step) - that is, until no unverified nodes are separated by a vertex cut of size at most $k$ from the nodes that were successfully verified. (This vertex cut can include successfully verified nodes. We note that in this context there is no longer a reason to iteratively remove nodes in the procedure that computes the trust policy $\left(\Pi_{k}^{*}\right)$ : because our goal is for all remaining nodes to be deemed legitimate, we simply need to check whether nodes are removed in the first iteration-if so, we need to verify additional nodes.)

\subsection{Optimally deciding which nodes to verify}

We now turn to the following optimization problem: how do we minimize the number of nodes that we verify before reaching the point where all the remaining nodes are deemed legitimate? To answer this question, we first note that, since there will be no incentive to create illegitimate nodes, we can assume that all nodes will in fact be legitimate. (This does not mean that we can afford to not do the verification, because if we did not, then there would be incentives to create illegitimate nodes again.) Hence, the problem becomes to find a minimal subset of nodes so that no other node is separated from these nodes by a vertex cut of size at most $k$ (which may include nodes in this subset)_or, equivalently, by Lemma 1, to find a minimal subset of nodes so that every other node is connected by $k+1$ vertex-disjoint paths to (distinct nodes in) this subset.

This problem is a special case of the source location problem. A polynomial-time algorithm for this problem is given in a paper by Nagamochi et al. [12]. They show that the problem has a matroidal property, as 
follows. Instead of thinking about minimizing the number of verified nodes, we can think about maximizing the number of unverified nodes. Say a subset $U \subseteq V$ is feasible if, for every $v \in U$, there exist $k+1$ vertex-disjoint (apart from $v$ ) paths to (distinct) nodes in $V \backslash U$.

Theorem 5 ([12]) The feasible sets satisfy the independence axioms of a matroid.

Finding an independent set of maximum size in a matroid is easy: start with an empty set, and attempt to include the elements one at a time, being careful not to violate the independence property. In the context of trying to find a minimum-size set of nodes to verify, this corresponds to starting with the set of all nodes, and attempting to exclude the nodes one at a time, being careful that it will still result in all the excluded nodes being deemed legitimate. The following lemma states that to check the latter, we only need to consider the current node:

Lemma 4 Suppose $S \subseteq V$ is such that from every $u \in V-S$, there exist $k+1$ vertex-disjoint paths to (distinct nodes in) $S$, and suppose that for some $v, S-\{v\}$ does not have this property. Then, there do not exist $k+1$ vertex-disjoint paths from $v$ to (distinct nodes in) $S-\{v\}$.

This results in the following simple polynomial-time algorithm $\Phi_{k}$ for finding a minimum-size set of nodes to verify.

Definition $8 \Phi_{k}$ takes as input a graph $G=(V, E)$ and proceeds as follows to determine the nodes $S$ to verify:

1. Initialize $S \leftarrow V$.

2. For each node $v \in S$ : if there are $k+1$ vertex-disjoint paths from $S \backslash v$ to $v$, then remove $v$ from $S$.

3. Return $S$.

In Appendix B.2 we implement $\Phi_{k}$ and see what the number of trusted nodes needed is for different values of $k$ on some simulated social networks.

\section{Conclusions and Future Research}

From the above, it becomes clear that false-name-proofness, while achievable in social networking settings, does not come for free: we either cannot let all agents participate, or we must spend significant effort verifying identities. How severe these downsides are depends on the exact structure of the social network. If we have a sufficiently densely connected social network, then almost everyone can participate even when there are relatively few trusted identities, or, alternatively, we only need to verify a small number of identities to let everyone participate. But, is this likely to be the case in realistic social networks? In the appendix, we provide some results based on experimental simulation; future research may be devoted to obtaining analytical characterizations.

Future research may also be devoted to considering some changes in the basic model and their effect on our results. What happens if agents can decide to drop edges (that is, not declare friendships) for strategic reasons? What happens if agents can get other agents to link to their fake identities at a cost? Results here may be reminiscent of those obtained in existing models where additional identifiers can be obtained at a cost [14]. What happens when we can only verify a limited number of nodes and try to maximize the number of nodes deemed legitimate? 


\section{References}

[1] Réka Albert and Albert-László Barabási. Statistical mechanics of complex networks, 2001.

[2] R. Andersen, C. Borgs, J. Chayes, J. Hopcroft, V. Mirrokni, and S. Teng. Local computation of PageRank contributions. In 5th International Workshop of Algorithms and Models for the Web-Graph, 2007.

[3] Reid Andersen, Christian Borgs, Jennifer T. Chayes, John E. Hopcroft, Kamal Jain, Vahab S. Mirrokni, and Shang-Hua Teng. Robust PageRank and locally computable spam detection features. In AIRWeb, pages 69-76, 2008.

[4] Vincent Conitzer. Limited verification of identities to induce false-name-proofness. In TARK, pages 102-111, Brussels, Belgium, 2007.

[5] Vincent Conitzer. Anonymity-proof voting rules. In Proceedings of WINE, pages 295-306, Shanghai, China, 2008.

[6] Zoltán Gyöngyi, Pavel Berkhin, Hector Garcia-Molina, and Jan Pedersen. Link spam detection based on mass estimation. In Proceedings of the 32nd International Conference on Very Large Databases. ACM, 2006.

[7] Zoltán Gyöngyi, Hector Garcia-Molina, and Jan Pedersen. Combating web spam with TrustRank. In Proceedings of the 30th International Conference on Very Large Databases, pages 576-587. Morgan Kaufmann, 2004.

[8] Atsushi Iwasaki, Vincent Conitzer, Yoshifusa Omori, Yuko Sakurai, Taiki Todo, Mingyu Guo, and Makoto Yokoo. Worst-case efficiency ratio in false-name-proof combinatorial auction mechanisms. In Proceedings of AAMAS, Toronto, Canada, 2010.

[9] John M. Kleinberg. Navigation in a small world. Nature, 2000.

[10] Duncan R. Luce and Howard Raiffa. Games and decisions: introduction and critical survey, New York, 1957

[11] Karl Menger. Zur allgemeinen Kurventheorie. Fund. Math., 10:96-115, 1927.

[12] Hiroshi Nagamochi, Toshimasa Ishii, and Hiro Ito. Minimum cost source location problem with vertexconnectivity requirements in digraphs. Information Processing Letters, 80(6):287-293, 1993.

[13] R. Raj and V. Krishnan. Web spam detection with anti-trust rank. In 2nd International Worshop on Adversarial Information Retrieval on the Web, pages 381-389, 2006.

[14] Liad Wagman and Vincent Conitzer. Optimal false-name-proof voting rules with costly voting. In Proceedings of AAAI, pages 190-195, Chicago, IL, USA, 2008. Outstanding paper award.

[15] Makoto Yokoo, Yuko Sakurai, and Shigeo Matsubara. Robust combinatorial auction protocol against false-name bids. Artificial Intelligence, 130(2):167-181, 2001.

[16] Makoto Yokoo, Yuko Sakurai, and Shigeo Matsubara. The effect of false-name bids in combinatorial auctions: New fraud in Internet auctions. Games and Economic Behavior, 46(1):174-188, 2004.

[17] Haifeng Yu, Phillip B. Gibbons, Michael Kaminsky, and Feng Xiao. SybilLimit: A near-optimal social network defense against sybil attacks. IEEE/ACM Transactions on Networking (ToN), 2010. To appear. 
[18] Haifeng Yu, Michael Kaminsky, Phillip B. Gibbons, and Abraham Flaxman. SybilGuard: Defending against sybil attacks via social networks. IEEE/ACM Transactions on Networking (ToN), 16(3):576$589,2008$.

[19] Mark Zuckerberg. Voting begins on governing the Facebook site, 2009. http://blog.facebook.com/blog.php?post=76815337130.

\section{A Omitted figure and proofs}

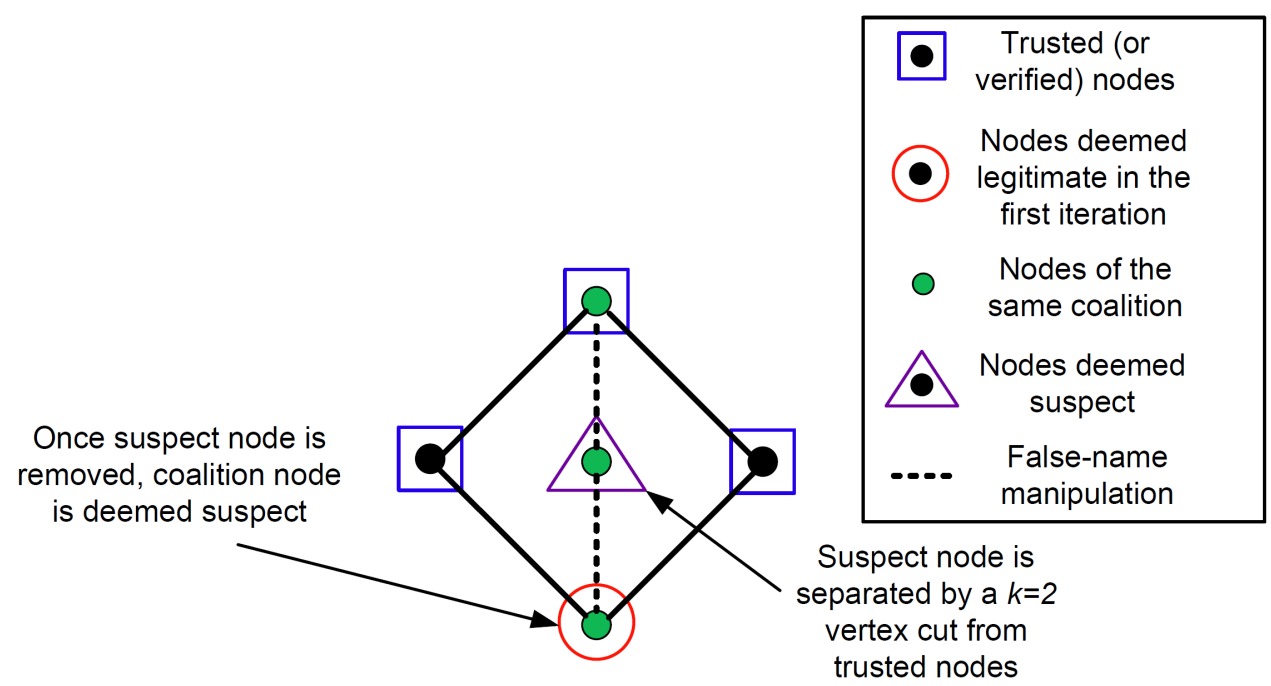

Figure 2: An example network when an iterative procedure is necessary. Again, the nodes correspond to identities (user accounts), and the edges correspond to (say) friendship relations between the identities. The mechanism designer, at this point for exogenous reasons, considers certain nodes "trusted" (marked by squares), that is, she is sure that they are not false names. The node marked with a triangle is separated from the trusted nodes by a vertex cut of size two in the first iteration. Furthermore, after removing this node (and its adjacent edges), the node marked with a circle is separated from the trusted nodes by a vertex cut of size two in the second iteration. As a result, these two nodes will be considered false nodes and both labeled suspect.

Theorem 1 If a suspicion policy $\Pi$ is $k$-robust, then it guarantees false-name-proofness for coalitions of size at most $k$.

Proof. We need to prove that, under the LIA assumption, it is a dominant strategy for each coalition to set $V_{S_{j}}^{f}=\emptyset$ and report truthfully. We note that which nodes in $V_{S_{j}}^{t} \cup V_{S_{j}}^{f}$ are deemed legitimate is unaffected by the actions of the other coalitions, due to the first condition for $k$-robustness. Combining this with the LIA assumption, it follows that the coalition $S_{j}$ learns nothing about the other coalitions' actions until the final outcome is announced (technically, regardless of the actions of the other coalitions, $S_{j}$ ends up in the same information set). Hence, $S_{j}$ can make the decision of what to report to $f$ at the same time as deciding what nodes and edges to create. 
Similarly, $S_{j}$ 's actions do not affect which nodes outside $V_{s_{j}}^{t} \cup V_{S_{j}}^{f}$ are deemed legitimate by $k$-robustness, and hence, again using the LIA assumption, $S_{j}$ 's actions also do not affect the preferences reported by other coalitions. Thus, the only effect that setting $V_{S_{j}}^{f} \neq \emptyset$ can have is that the coalition $S_{j}$ ends up with a different number of identifiers that are deemed legitimate; by the second condition for $k$-robustness, it can only result in the same or a lower number of identifiers that are deemed legitimate. Because $f$ satisfies $k$-voluntary participation, this cannot be helpful. Because $f$ is $k$-strategy-proof, it follows that it is a dominant strategy for $S_{j}$ to set $V_{S_{j}}^{f}=\emptyset$ and report truthfully.

Lemma 1 For an initially untrusted node $v$, the following two statements are equivalent.

1. $v$ is not separated from the initially trusted nodes by a vertex cut of size at most $k$ (which may include initially trusted nodes).

2. There exist $k+1$ vertex-disjoint paths from (distinct) initially trusted nodes to $v$.

Proof. We modify the social network graph by creating a new node $s$ and connecting it to every one of the initially trusted nodes. According to Menger's theorem [11], $s$ and $v$ are not separated by a vertex cut of size at most $k$ if and only if there exist at least $k+1$ vertex-disjoint paths between $s$ and $v$. The lemma follows immediately.

Lemma 2 The class $\Pi_{k}$ consists of a singleton element $\Pi_{k}^{*}$, i.e., $\Pi_{k}=\left\{\Pi_{k}^{*}\right\}$.

Proof. We first note the following monotonicity property: if a node $v$ is separated from all the trusted nodes by a vertex cut of size at most $k$, then this remains the case for $v$ after we remove any subset of the other nodes in the graph. Thus, if we use a policy in $\Pi_{k}$, and a node becomes separated from the trusted nodes by a vertex cut of size at most $k$ at some point, then the policy must remove it eventually.

Let $\Pi_{k}^{\prime} \in \Pi_{k}$; we need to show that $\Pi_{k}^{\prime}=\Pi_{k}^{*}$. We first prove that any node $v$ labeled suspect by $\Pi_{k}^{*}$ will eventually be eliminated by $\Pi_{k}^{\prime}$, by induction on the iteration in which $v$ is eliminated by $\Pi_{k}^{*}$. In the base case (the first iteration), $v$ is separated from the trusted nodes by a vertex cut of size at most $k$ from the beginning, and so $\Pi_{k}^{\prime}$ must eventually remove it.

Suppose that we have proven the claim for the case where $v$ is eliminated in or before the $(t-1)$ th iteration of $\Pi_{k}^{*}$; we must show it holds for the case where $v$ is eliminated in the $t$ th iteration. By the induction assumption, all nodes eliminated in earlier iterations by $\Pi_{k}^{*}$ must eventually be eliminated by $\Pi_{k}^{\prime}$. We know that after all these nodes (and potentially others) are eliminated by $\Pi_{k}^{\prime}, v$ is separated from the trusted nodes by a vertex cut of size at most $k$-otherwise, $v$ would not be eliminated in the $t$ th iteration by $\Pi_{k}^{*}$. It follows that $\Pi_{k}^{\prime}$ must eventually remove $v$.

Finally, we prove that all nodes deemed legitimate by $\Pi_{k}^{*}$ are also deemed legitimate by $\Pi_{k}^{\prime}$. Assume for the sake of contradiction that there is a nonempty set of nodes that are deemed suspect by $\Pi_{k}^{\prime}$ but legitimate by $\Pi_{k}^{*}$; let $t$ be the first iteration in which $\Pi_{k}^{\prime}$ removes such a node, and let $v$ be one of these nodes eliminated in iteration $t$. We know that at the beginning of iteration $t$ in $\Pi_{k}^{\prime}$, none of the nodes deemed legitimate by $\Pi_{k}^{*}$ have been eliminated yet; hence, the graph at the beginning of iteration $t$ in $\Pi_{k}^{\prime}$ is a supergraph of the one that remains at the end of $\Pi_{k}^{*}$. But in the latter graph, $v$ is not separated from the trusted nodes by a vertex cut of size at most $k$ (otherwise, $\Pi_{k}^{*}$ would not have converged yet); and so the same must be the case in the former (super)graph as well. But then, $v$ cannot be eliminated in the $t$ th iteration of $\Pi_{k}^{\prime}$, and we have the desired contradiction.

Lemma 3 Let $G=(V, E)$ be a graph and let $T \subseteq V$ be the trusted nodes. Let $G^{\prime}$ be a graph that is obtained from $G$ by adding additional nodes $V^{\prime}$ and additional edges $E^{\prime}$ that each have at least one endpoint in $V^{\prime}$-in such a way that every node in $V^{\prime}$ is separated from $T$ by a vertex cut of size at most $k$. Then, applying $\Pi_{k}^{*}$ to $G^{\prime}=\left(V \cup V^{\prime}, E \cup E^{\prime}\right)$ and $T$ results in the same nodes being deemed legitimate as applying $\Pi_{k}^{*}$ to $G$ and $T$. 
Proof. Consider a suspicion policy $\Pi_{k}^{\prime}$ (applied to $G^{\prime}$ and $T$ ) that does the following: in the first iteration, it removes $V^{\prime}$; in the remaining iterations (on $G$ ), it removes the same nodes as $\Pi_{k}^{*}$. We observe that $\Pi_{k}^{\prime} \in \Pi_{k}$, because by assumption, at the beginning every node in $V^{\prime}$ is separated from $T$ by a vertex cut of size at most $k$, and after that $\Pi_{k}^{\prime}$ behaves as $\Pi_{k}^{*}$. Hence, by Lemma 2, applying $\Pi_{k}^{*}$ to $G^{\prime}$ and $T$ will result in the same nodes being deemed legitimate as applying $\Pi_{k}^{\prime}$ to $G^{\prime}$ and $T$. Moreover, by the construction of $\Pi_{k}^{\prime}$, applying $\Pi_{k}^{\prime}$ to $G^{\prime}$ and $T$ will result in the same nodes being deemed legitimate as applying $\Pi_{k}^{*}$ to $G$ and $T$. The result follows.

Theorem $2 \Pi_{k}^{*}$ is k-robust (and hence, by Theorem 1, guarantees false-name-proofness for coalitions of size at most $k$ ). Moreover, under $\Pi_{k}^{*}$, a coalition $S_{j}$ 's actions also do not affect which of its own legitimate nodes $V_{S_{j}}^{t}$ are deemed legitimate. Finally, $\Pi_{k}^{*}$ is guaranteed to label every illegitimate node as suspect.

Proof. First, we show that the actions of coalition $S_{j}$ do not affect which of the other coalitions' identifiers $\left(V \backslash\left(V_{S_{j}}^{t} \cup V_{S_{j}}^{f}\right)\right)$ are deemed legitimate (the first part of $k$-robustness) - and that, in fact, they also do not affect which of the nodes in $V_{S_{j}}^{t}$ are deemed legitimate. This follows from Lemma 3: if we let $G$ be the graph that results when $S_{j}$ does not create any false names, and $G^{\prime}$ be a graph that results when it does, then $G$ and $G^{\prime}$ satisfy the conditions of the lemma (because $V_{S_{j}}^{t}$ is a vertex cut of size at most $k$ that separates the extra nodes $V_{S_{j}}^{f}$ ) in $G^{\prime}$ from the trusted nodes from $T$ ) and hence they result in the same nodes being deemed legitimate in the end.

Second, we show that for any coalition $S_{j}$, the number of identifiers in $V_{S_{j}}^{t} \cup V_{S_{j}}^{f}$ that are deemed legitimate is maximized by setting $V_{S_{j}}^{f}=\emptyset$ (the second part of $k$-robustness). We first observe that all of the nodes in $V_{S_{j}}^{f}$ will be labeled as suspect by $\Pi_{k}^{*}$ (corresponding to the last statement in the theorem). This is because $V_{S_{j}}^{t}$ is a vertex cut of size at most $k$ that separates the nodes in $V_{S_{j}}^{f}$ from the trusted nodes, so all of $V_{S_{j}}^{f}$ will be eliminated in the first iteration. Moreover, because from the first part of the proof, $S_{j}$ 's actions do not affect which of the nodes in $V_{S_{j}}^{t}$ are deemed legitimate, it follows that the number of identifiers in $V_{S_{j}}^{t} \cup V_{S_{j}}^{f}$ that are deemed legitimate is maximized by setting $V_{S_{j}}^{f}=\emptyset$.

Theorem 3 Let $\Pi^{\prime}$ be a suspicion policy that (1) is k-robust, (2) is such that a coalition $S_{j}$ 's actions also do not affect which of its own legitimate nodes $V_{S_{j}}^{t}$ are deemed legitimate, and (3) is guaranteed to label every illegitimate node as suspect. Then, if $\Pi_{k}^{*}$ labels a node as suspect, then so must $\Pi^{\prime}$.

Proof. Consider a vertex cut of size at most $k$ that separates some nonempty set of nodes $V^{\prime}$ from $T$. Then, by property (3), $\Pi^{\prime}$ must label all nodes in $V^{\prime}$ as suspect, because the vertex cut may correspond to a coalition that artificially created all of $V^{\prime}$. Let $G^{\prime}$ be the graph that results from removing $V^{\prime}$ from $G$. Then, by properties (1) and (2), $\Pi^{\prime}$ must deem the same nodes legitimate when given $\left(G^{\prime}, T\right)$ as it would when given $(G, T)$-otherwise, the coalition corresponding to the vertex cut could affect which of the other coalitions' nodes are labeled suspect, or which of its own legitimate nodes are labeled suspect, by artificially creating (exactly) the nodes in $V^{\prime}$ (when $G^{\prime}$ is the true graph).

Now, consider a policy $\Pi_{k}^{\prime} \in \Pi_{k}$ that in each iteration identifies a single vertex cut of size at most $k$ that separates some nonempty set of nodes $V^{\prime}$ from $T$, and then removes these nodes $V^{\prime}$. By iteratively applying the reasoning above, it follows that any node eliminated by $\Pi_{k}^{\prime}$ must also be deemed suspect by $\Pi^{\prime}$. But by Lemma $2, \Pi_{k}^{\prime}$ and $\Pi_{k}^{*}$ are the same policy. It follows that if $\Pi_{k}^{*}$ labels a node as suspect, then so must $\Pi^{\prime}$.

Theorem 4 Given $G=(V, E)$ and $T \subseteq V$, we can determine in polynomial time which nodes are not separated from $T$ by a vertex cut of size at most $k$. As the number of iterations of $\Pi_{k}^{*}$ is bounded by $|V|$, we can run $\Pi_{k}^{*}$ in polynomial time. 
Proof. For each node $v \notin T$, we solve a maximum flow problem with capacities on the nodes. As in the proof of Lemma 1, we modify the social network graph by creating a new node $s$ and connecting it to every one of the nodes in $T . s$ will serve as the source, and $v$ as the sink. Every node (other than the source and the sink) has a capacity of 1 . By the integrality property of network flows, the maximum flow will be at least $k+1$ if and only if there exist $k+1$ vertex-disjoint paths from (distinct) nodes in $T$ to $v$, which by Lemma 1 is the case if and only if $v$ is not separated from $T$ by a vertex cut of size at most $k$ (which may include nodes in $T)$.

Lemma 4 Suppose $S \subseteq V$ is such that from every $u \in V-S$, there exist $k+1$ vertex-disjoint paths to (distinct nodes in) $S$, and suppose that for some $v, S-\{v\}$ does not have this property. Then, there do not exist $k+1$ vertex-disjoint paths from $v$ to (distinct nodes in) $S-\{v\}$.

Proof. Suppose $v^{\prime}$ is a node from which there are $k+1$ vertex-disjoint paths to (distinct nodes in) $S$, but not to $S-\{v\}$. By Lemma 1, there exists a vertex cut of size at most $k$ separating $v^{\prime}$ from $S-\{v\}$ (this vertex cut possibly includes nodes in $S-\{v\}$ ). $v$ must lie on the same side of this cut as $v^{\prime}$, because otherwise the vertex cut would also separate $v^{\prime}$ from $S$. It follows that the vertex cut also separates $v$ from $S-\{v\}$.

\section{B Experimental results}

To evaluate how many nodes will be deemed legitimate, or how many nodes we need to verify, in "typical" social networks, we conducted a series of experiments on randomly generated graphs. To generate these graphs we used two models, Kleinberg's model for navigable small world networks [9] and the BarabásiAlbert model [1] for generating scale-free networks.

We construct random social networks with Kleinberg's model as follows (labeled "Klein" in the following graphs). Take a square grid and connect each node to its neighbors. Next, for each node $v$, add one additional connection to a random node $v^{\prime}\left(v^{\prime} \neq v\right)$ in the graph, with probability proportional to $1 /\left(\operatorname{distance}\left(v, v^{\prime}\right)\right)$.

We construct random social networks with the Barabási-Albert model as follows. Start with a small seed graph (we start with a small grid of 9 nodes). We add the remaining nodes one by one. For each node $v$ we add, we connect it to 3 (BA-6) or 5 (BA-10) of the existing nodes. To choose which nodes $v$ will be connected to, we randomize over the existing nodes, but the probablity of picking a specific node $v^{\prime}$ is proportional to the number of neighbors that $v^{\prime}$ has (this means that nodes that already have a large number of neighbors have a higher probability of receiving more neighbors).

We used the JUNG libary (Java Universal Network/Graph Framework) and CPLEX in our implementation. Due to running time and memory constraints faced by this methodology, the largest graphs we consider are of 2025 nodes (and in fact we will only present results on graphs of 2025 nodes). In the results in the following two subsections, the numbers are averages taken over 20 graphs.

\section{B.1 Results for the exogenous case}

In this subsection, we present our results when some nodes in the graph are chosen at random to be trusted nodes. In our implementation, we took advantage of Lemma 2 to significantly reduce the running time of the experiment, based on the fact that if a node is eliminated when only $k+1$ vertex-disjoint paths are necessary for legitimacy, it will still be eliminated when we require $k+2$ paths. Thus, we can start the step of the experiment where $k+2$ paths are necessary by removing all of the nodes eliminated at the end of of the step where $k+1$ paths are necessary, and still get the same solution that we would have obtained by running the algorithm from the begining.

As shown in Figure 3, for small $k$ the number of nodes deemed legitimate is very high, but as $k$ grows there is eventually a sharp dropoff for every one of the random graph generation models (though at different 
points for the different models). This dropoff can be partially explained by the fact that eventually, there will be many nodes that have fewer than $k+1$ neighbors (as shown in Figure 3(c)), and these nodes will be considered suspect unless they happen to be trusted themselves. However, the number of nodes deemed legitimate does drop faster than the number of nodes with at least $k+1$ neighbors. This could be due to, for example, the iterated removal of nodes-once some nodes are removed, others may start to be removed as well.
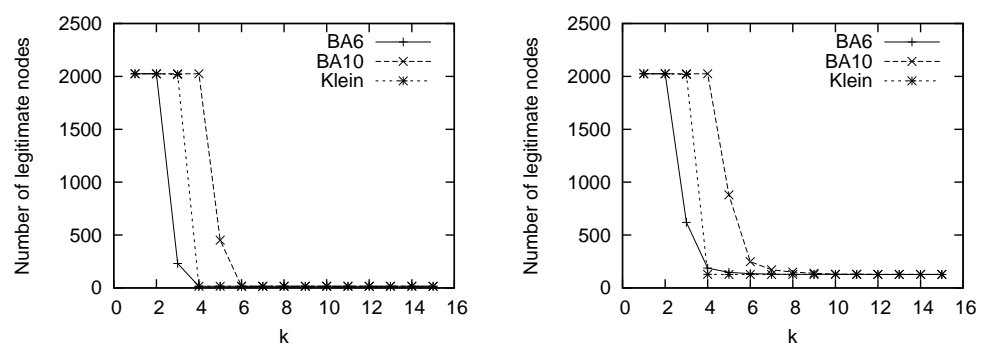

(a) Number of nodes deemed legitimate with (b) Number of nodes deemed legitimate with 16 random trusted nodes.

128 random trusted nodes.

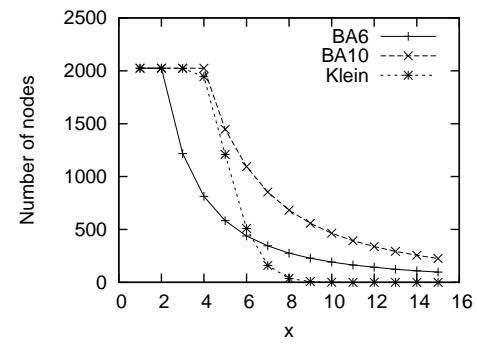

(c) Number of nodes with degree $>x$

Figure 3: Results for exogenously trusted nodes.

\section{B.2 Results for the endogenous case}

In this subsection, we present our results for the case where no nodes are initially trusted, and we choose a subset of nodes to verify so that all nodes will be deemed legitimate. Of course, every node with degree lower than $k+1$ must be verified. Our results in Figure 4 show that that is basically all we need to do: in this case, the number of nodes that need to be verified very closely matches the number of nodes with degree $\leq k$ for both types of social network. (Though it is difficult to see, the graphs are in fact very slightly different.) 


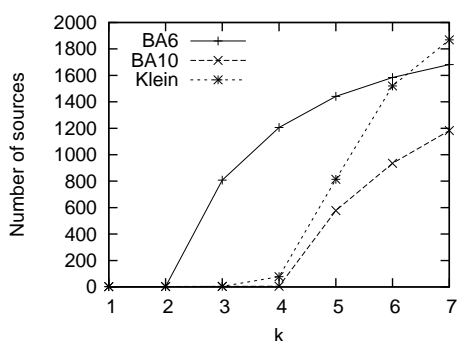

(a) Number of nodes that must be verified for all nodes to be deemed legitimate

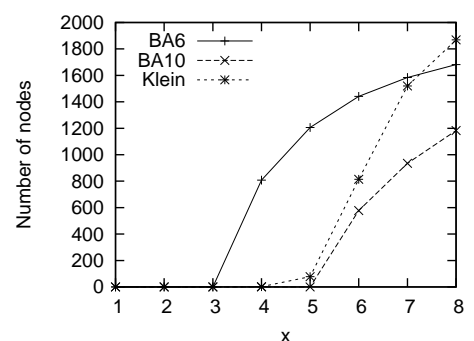

(b) Number of nodes with degree $\leq x$

Figure 4: Results for verification (endogenous trust). 